\title{
THE HEPARANASE INHIBITOR (SULODEXIDE) DECREASES URINE GLYCOSAMINOGLYCAN EXCRETION AND MITIGATES FUNCTIONAL AND HISTOLOGICAL RENAL DAMAGES IN DIABETIC RATS
}

\author{
S. Roshan-Milani' ${ }^{2,3}$, J. Khalilpour ${ }^{2}$, A. Abdollahzade Fard ${ }^{1,2}$ \\ ${ }^{1}$ Nephrology and Kidney Transplant Research Center, Urmia University of Medical Sciences, - Urmia, Iran \\ ${ }^{2}$ Departments of Physiology, Faculty of Medicine, Urmia University of Medical Sciences, - Urmia, Iran \\ ${ }^{3}$ Neurophysiology Research Center, Urmia University of Medical Sciences - Urmia, Iran
}

\begin{abstract}
Background/objectives: Recent data suggest a role for heparanase in several proteinuric conditions. An increased glomerular heparanase expression is associated with loss of heparan sulfate in the glomerular basement membrane (GBM). The aim of the present study was to investigate the renal effects of heparanase inhibition in a diabetic experimental model. Methods: Fifteen male Wistar rats $(230 \pm 20 \mathrm{~g})$ were divided into three groups: 1) controls, 2) diabetics (STZ, $50 \mathrm{mg} / \mathrm{kg}$, dissolved in saline, ip), 3) diabetics + heparanase inhibitor (Sulodexide 1/5 mg/kg per day, gavage). The treatment started on the 21st day, for 21 consecutive days. The rats were kept individually in a metabolic cage (8 AM-2 PM) and urine samples were collected on the 21st and 42nd day. At study end blood, urine and tissue samples were collected for biochemical (blood BUN and Cr, urine GAG and Protein) and histological analyses. Results: The results of this study showed that the heparanase inhibitor (sulodexide) significantly decreased urine GAG and protein excretion, urine protein/creatinine ratio and serum BUN and $\mathrm{Cr}$ in streptozotocin-induced DN in the rats. Pathological changes were significantly alleviated in the $D N$ rats having received the heparanase inhibitor (sulodexide). Conclusion: Our data suggest that the heparanase inhibitor (sulodexide) is able to protect against functional and histopathological injury in DN.
\end{abstract}

Key words: Heparanase, diabetic nephropathy, sulodexide, urine protein, urine glycosaminoglycan

Corresponding author: Amin Abdollahzade Fard, Department of Physiology, Faculty of Medicine, Urmia University of Medical Sciences, Urmia, Iran, Tel: +98(44)32770698, Fax: +98(44)32780800, e-mail: af.amin@yahoo.com \& e-mail: abdollahzade.a@umsu.ac.ir

\section{INTRODUCTION}

$\mathrm{D}$ iabetic nephropathy (DN) is a major lifethreatening complication of diabetes, that is the leading cause of end-stage renal disease in developed countries [1, 2]. Abnormal perm-selectivity of the glomerular basement membrane (GBM) plays an important role in the pathogenesis of DN [3,
4]. The glomerular basement membrane (GBM) consists mainly of laminin, type IV collagen, and heparan sulfate proteoglycans (HSPGs). Heparan sulfate proteoglycans (HSPGs) are abundant in the extracellular matrices (ECMs) including basement membranes, and consist of diverse core polypeptides and the HS [5, 6]. In patients with DN, loss of Heparan sulfate proteoglycans from theglomerular extracellu- 
lar matrices has been reported [7]. Heparan sulfate maintains the mechanical integrity of the glomerular basement membranes. Direct heparitinase digestion by the heparitinase existing in glomerular basement membranes results in a loss of membrane function [8]. Heparanase, an endoglycosidase, is the predominant enzyme that specifically cleaves heparan sulfate, the main polysaccharide of the GBM $[3,9]$. In patients with DN, loss of HSPG in glomerular extracellular matrices of diabetic kidney has been reported and this reduction was associated with increased glomerular expression of heparanase [7]. An increase in urinary heparanase activity was observed in both type 1 and type 2 diabetic patients with proteinuria [10]. In the early phase of human Diabetic nephropathy, there is a urinary loss of albumin, consistent with a loss of heparan sulfates in the glomerular basement membrane [11]. Therefore, loss of heparan sulfates in the GBM results in a decrease of the anionic charge barrier and may possibly be resulting in progression of proteinuria and deterioration of renal function in the DN [11, 12].

Well known heparanase- 1 inhibitors are heparin(s) and sulodexide, a low-molecular weight heparin / dermatan sulphate blend, which is effective in the treatment of DN. Sulodexide is a mixture of glycosaminoglycans composed of a $80 \%$ fast mobility heparin fraction and $20 \%$ dermatan sulfate. Sulodexide differs from other glycosaminoglycans, like heparin, because does not have anti-coagulation properties when given orally and have a longer half-life [13, 14]. Treatment with sulodexide has been shown to reduce proteinuria in patients with $D N[15,16]$. However, data from a recent controlled trial showed negative results [17]. As mentioned above the effect of sulodexide on urine protein and glycosaminoglycan excretion is not exactly clear. In the present study, we investigated the effect of a heparanase inhibitor (sulodexide) on urine excretion of protein and glycosaminoglycan and on renal histology in type I diabetic nephropathy.

\section{MATERIAL AND METHODS}

Experimental design: Fifteen male 10 week-old Wistar rats weighing $230 \pm 20 \mathrm{~g}$ were purchased by the animal house of the Urmia University of Medical Sciences, Urmia, Iran. All procedures on the animals were conducted in accordance with the Principles of Laboratory Animal Care and approved by the Ethical Committee of the Urmia University of Medical Sciences. The animals were housed under standard conditions $\left(21 \pm 2^{\circ} \mathrm{C}\right.$ and $12 / 12 \mathrm{hr}$ light/dark cycle) . The animals were fed with normal rat diet and water.
Animal grouping: The animals were divided into three groups (five animals each): Group 1 - healthy controls ( $0.5 \mathrm{ml}$ of NS, daily, gavage), Group 2 - diabetic group, and Group 3- diabetic group treated with sulodexide (1/5 $\mathrm{mg} / \mathrm{kg}$; daily, gavage). Diabetes was induced by a single dose of streptozotocin (STZ, 50 $\mathrm{mg} / \mathrm{kg} \mathrm{BW}$, dissolved in saline. ip). Five days after the STZ injection, blood glucose levels were determined with a glucose strip test and a glucometer. Rats with blood glucose levels above $250 \mathrm{mg} / \mathrm{dL}$ were defined as the diabetic animals. The treatment started on the 21st day after the STZ injection since we found a significant increase in urine protein excretion (UPE) rate in the diabetic rats in comparison with the control rats. The treatment was continued daily for three weeks.

For twenty one days after the STZ injection, the rats were kept individually in metabolic cages (8 AM-2 $\mathrm{PM}$ ) and urine samples were collected for 6-h measurement of UPE [18] and urine creatinine. The observations revealed a significant increase in the UPE rate in the diabetic rats in comparison with the control rats and the animals were considered nephropathic.

At the end of the sixth week, 6-h urine samples were collected for biochemical analysis. Then, five rats from each subgroup were sacrificed under ether anesthesia. Moreover, blood samples were collected by cardiac puncture and tissue samples were stored in formalin.

Biochemical analyses: Serum BUN and creatinine were measured using an auto-analyzer. Additionally, a 6 h urine sample was collected for measurement of BUN, Cr, UPE and glycosaminoglycan (GAG).

Urine protein excretion [18] was determined by a Pars Azmon kit (Iran). Further, UPE was measured by quantitative reaction with bromocresol green [28] using bovine serum albumin as standard. A sample volume of $10 \mu \mathrm{l}$ and a validated standard were mixed separately with $1 \mathrm{ml}$ of bromocresol green and then the absorbance was read at $625 \mathrm{~nm}$.

Urinary GAG in the 6-hour urine samples was measured spectrophotometrically at a wavelength of 520 $\mathrm{nm}$, with a colorimetric method described by Jong, [29] using 1.9 dimethyleneblue and bovine kidney heparan sulfate as standard (Sigma Cat No H7640).

Histological study: At the end of the study the right kidney was removed and stored in $10 \%$ formaldehyde. Kidney sections ( $5 \mathrm{~mm}$ ) were stained with periodic acid-Schiff (PAS) and Masson's trichrome (MTC) for histologic and morphometric analysis. Mesangial matrix accumulation was assessed by PAS-positive staining in nuclei-free areas of the mesangium. The mesangial matrix was evaluated in 30 randomly selected glom- 
eruli and scored in a blinded way on a scale of 0 to 4 , where $0=0-5 \%, 1=0.5-25 \%, 2=25-50 \%, 3=50-$ $75 \%$, and $4=>75 \%$ deposition. The scores revealed variations in the extent rather than in the intensity of staining [30]. The "sclerotic index" referred to the mean score. Collagen deposition was measured by Masson's trichrome staining of 30 glomeruli, scored in a blinded way using the above-mentioned system and expressed as an arbitrary unit [30, 31]. Then, it was expressed as a mean sclerotic index and glomerular collagen staining score for each group.

Statistical analysis: Data were expressed as means \pm SDs. Statistical significance of differences was assessed by one-way ANOVA on the SPSS (Version 18; SPSS Inc., Chicago, USA) followed by Tukey's test. A p-value of less than 0.05 was considered statistically significant. Linear regression analyses were applied to evaluate the relationship between two variables.

\section{RESULTS}

Effects of Sulodexide administration on serum BUN, Creatinine, $\mathrm{K}$ and glucose

The results show significantly increased levels of serum BUN, Cr, $\mathrm{K}$ and glucose of the DN rats in comparison with the control (Table 1 \& Figure 1, $p$ $<0.001$ ). However, the administration of sulodexide in the DN decreased serum BUN, Cr, K and these changes were statistically significant (Table 1, p < 0.01 ). Moreover, there was no significant difference in the serum concentration of glucose between the DN and the sulodexide treated groups (Figure 1). All together, these data suggest that sulodexide was able to protect against the DN injury but did not significantly affect the changes of serum glucose.

Table 1. Serum \& Urime biochemical analises in the study groups

\begin{tabular}{|l|l|l|l|}
\hline Parameters & Control & DN & DN+Sul \\
\hline Serum & & & \\
\hline Creatinine $(\mathrm{mg} / \mathrm{dl})$ & $0.49 \pm 0.2$ & $1.1 \pm 0.28^{*}$ & $0.65 \pm 15 \dagger$ \\
\hline BUN $(\mathrm{mg} / \mathrm{dl})$ & $13.2 \pm 2.6$ & $27.3 \pm 4.9^{*}$ & $19.1 \pm 2.4 \dagger$ \\
\hline $\mathrm{K}(\mathrm{meq} / \mathrm{L})$ & $4.8 \pm 0.5$ & $5.8 \pm 0.7^{*}$ & $4.4 \pm 0.5 \dagger$ \\
\hline Urine & & & \\
\hline Creatinine $(\mathrm{mg} / \mathrm{dl})$ & $32.7 \pm 5.1$ & $25.9 \pm 5.7$ & $29.8 \pm 6.8$ \\
\hline Uprot.21 $(\mathrm{mg} / 6 \mathrm{~h})$ & $9.1 \pm 2.1$ & $55 \pm 12.4^{*}$ & $49.6 \pm 18.3^{*}$ \\
\hline Uprot.42 $(\mathrm{mg} / 6 \mathrm{~h})$ & $10.2 \pm 4.2$ & $69 \pm 26.4^{*}$ & $21 \pm 8.6 \dagger$ \\
\hline
\end{tabular}

The values show as Mean $\pm S D, D N=$ (diabetic nephropathy), Sul = (Sulodexide), Uprot.21 \& $42=$ (urine protein at 21,42 th day), * and + Show the significance in comparison with control and DN group respectively $(p<0.05)$

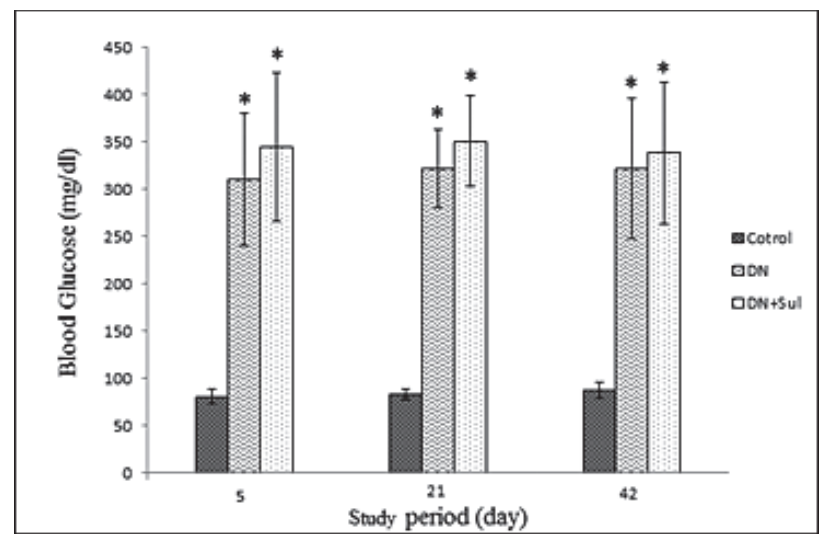

Fig. 1. Blood glucose in the 5, 21, 42th day of the study period. The values are shown as Mean \pm SD, DN = (Diabetic Nephropathy) and Sul = (Sulodexide); ${ }^{\text {* }}$ shows the significance in comparison with the control $(p<0.001)$

Effects of Sulodexide on urine protein excretion and protein-creatinine ratio (PCR)

Three weeks after streptozotocin administration, urinary 6-hour protein excretion level was 9.1 $\pm 2.1,55$ \pm 12.4 and $49.6 \pm 18.3 \mathrm{mg} / 6 \mathrm{~h}$ in the healthy controls, diabetics and treated diabetics, respectively. Urinary protein excretion increased significantly in the diabetic rats $(p<0.01)$. Moreover, urinary 6-hour albumin excretion level remained high over time in the DN in comparison with the control group $(p<$ $0.001)$. However, the administration of sulodexide significantly reduced the urine 6 -h protein excretion $(p<0.001)$ in the DN in comparison with the untreated DN group (Table 1). The results showed a positive effect of sulodexide on the STZ-induced DN in the rats, which was evidenced by the significant decrease $(p<0.001)$ in the urinary protein/creatinine ratio (Figure 2).

\section{Effects of Sulodexide on urine glycosaminoglycan GAG}

At the end of the six study weeks, 6-hour urinary glycosaminoglycan (GAG) levels were significantly higher in the DN rats in comparison with the healthy controls (133.4 \pm 20.2 and $44.2 \pm 6.3$ respectively; $p$ $<0.001)$. However, the sulodexide treated group had a significantly lower GAG excretion $(97 \pm 16.4)$ than the untreated DN rats $(p<0.01$, Figure 3$)$.

Correlation of urinary protein with urinary glycosaminoglycan excretion:

Urinary protein excretion was significantly correlated with urinary glycosaminoglycan excretion $(r=0.88, p$ $<0.001$ ) in all the study groups (Figure 4).

Effects of Sulodexide on morphological changes after diabetic nephropathy (DN) injury:

Diabetic nephropathy led to increased glomerular surface area, mesangial expansion, thickening of the 
GBM and Bowman's capsule, and increased deposition of matrix proteins within the mesangial matrix (Figure 5 and 6). The "sclerotic index", which reflects glomerular matrix accumulation, increased in DN rats in comparison with the controls $(2.75 \pm 0.19$ vs 0.23 \pm 0.07 , three weeks after onset of albuminuria, $P<$ 0.001 ), but sulodexide treatment significantly reduced

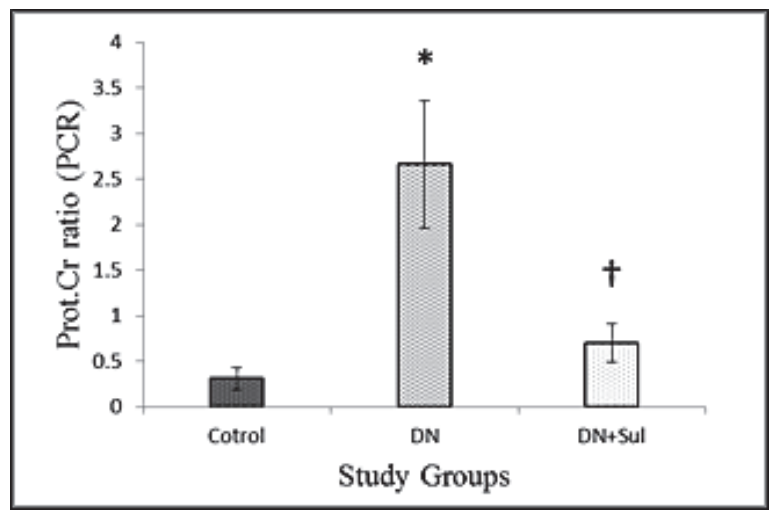

Fig. 2. The urine protein/creantinine ratio; the values are shown as Mean \pm SD, DN = (Diabetic Nephropathy) and Sul = (Sulodexide); ${ }^{*}$ and $†$ show the significance in comparison with the control and DN group, respectively $(p<0.001)$
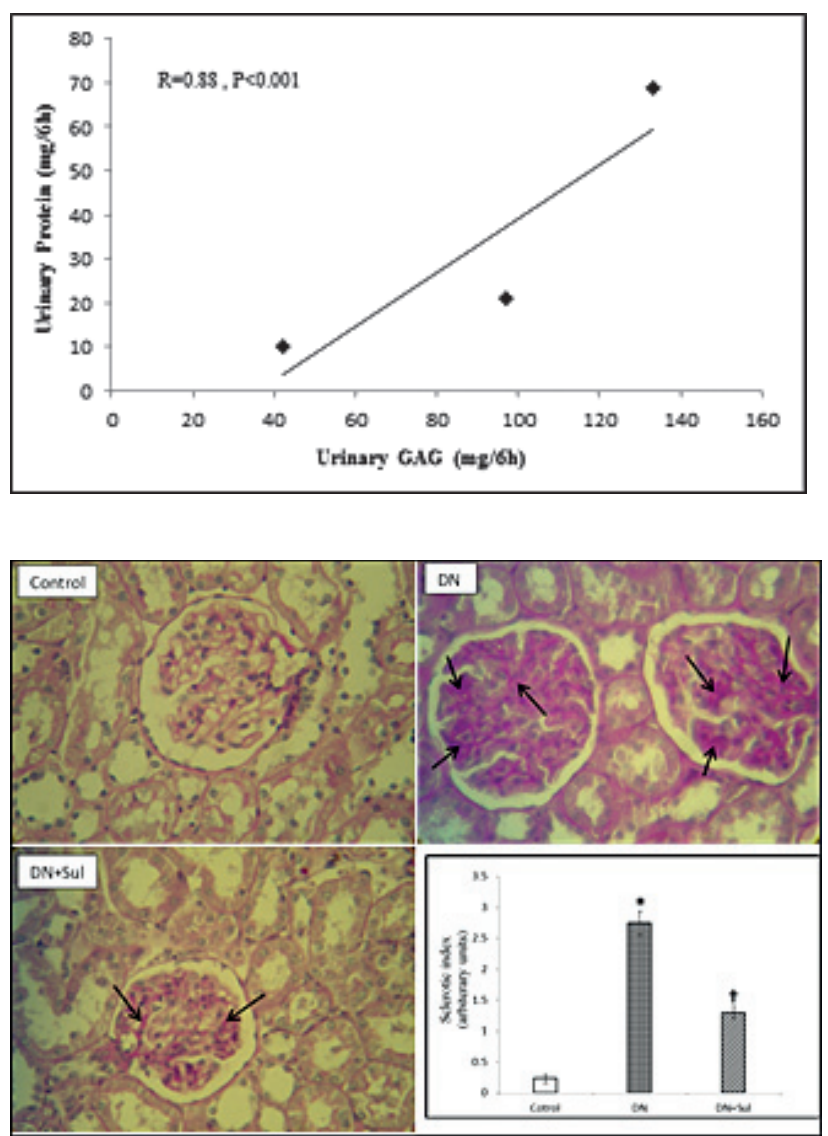

Fig. 5. Photomicrographs of PAS staining of renal tissues; the $D N=$ (Diabetic Nephropathy) and Sul = Sulodexide; arrows show the mesangial matrix accumulation in nuclei-free areas of the mesangium in glomeruli. The "sclerotic index" referred to the mean \pm SE score. The scores reflected variations in the extent rather than intensity of staining. ${ }^{*}$ and + show the significance in comparison with the control and DN group, respectively $(p<0.001)$. Magnification: 400x
$(1.3 \pm 0.1, p<0.001)$ the sclerotic index (Figure 5). Collagen deposition in the glomerulus was markedly reduced in the $\mathrm{DN}$ following the sulodexide treatment $(2.29 \pm 0.15$ vs $0.83 \pm 0.09, p<0.001)$ (Figure 6). As shown in Figures 5 and 6 , the pathological changes due to diabetic nephropathy were significantly alleviated in the sulodexide treated $\mathrm{DN}$ rats.

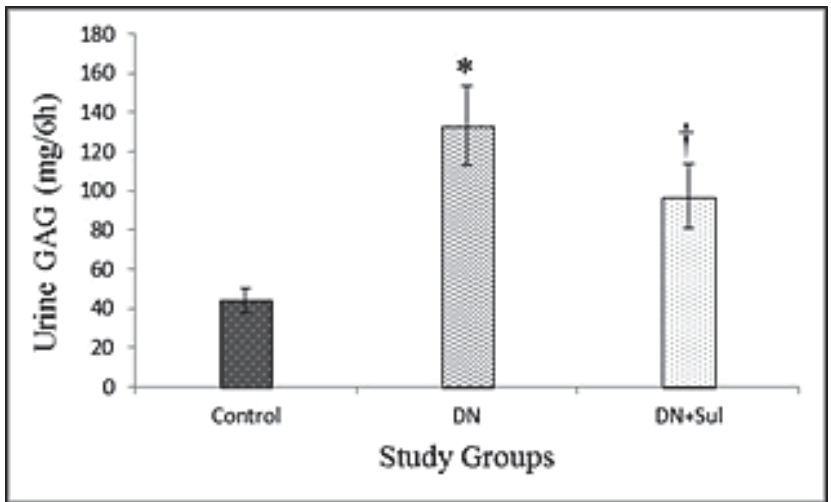

Fig. 3. The urine glycosaminoglycan (GAG); the values are shown as Mean \pm SD, DN = (Diabetic Nephropathy) and Sul = (Sulodexide); ${ }^{*}$ and $\dagger$ show the significance in comparison with the control and DN group, respectively $(p<0.01)$

Fig. 4. Linear regression curves representing urinary protein and glycosaminoglycan in the normal and diabetic rats $(r=0.88$ and $\mathrm{p}<0.001)$

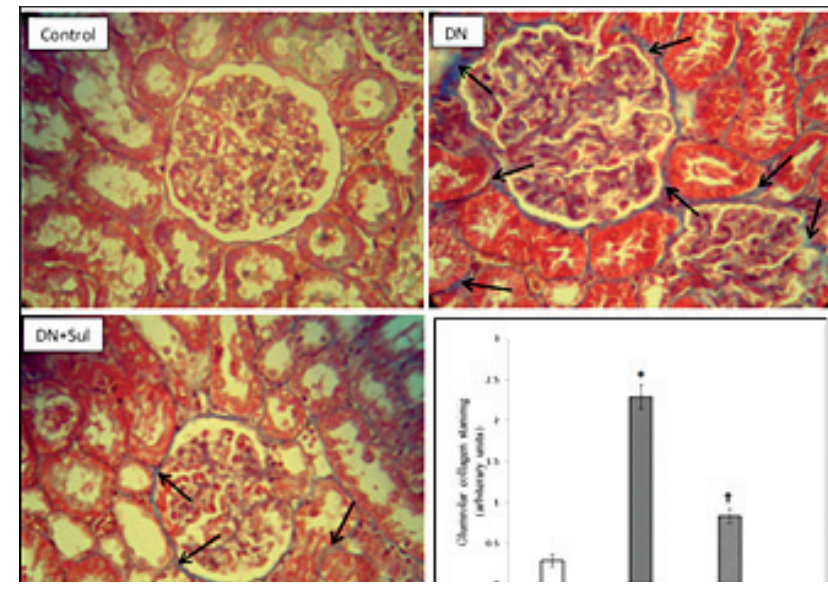

Fig. 6. Photomicrographs of Masson trichrome (MTC) staining of renal tissues. The DN = (Diabetic Nephropathy) and Sul = Sulodexide; arrows show the collagen deposition. The "glomerular collagen deposition" referred to the mean \pm SE score. * and † show the significance in comparison with the control and DN group, respectively $(p<0.001)$. Magnification: $400 x$ 


\section{DISCUSSION}

In the present study after STZ administration Wistar rats developed diabetes mellitus then pathological urine glycosaminoglycan and proteinuria excretion and also impaired kidney function. Rats with diabetic nephropathy showed predominantly increased glomerular surface area, mesangial expansion, thickening of the GBM and Bowman's capsule, and increased deposition of matrix proteins within the mesangial matrix but relatively mild tubulointerstitial changes. Our results are consistent with previous studies $[19,20]$. Over the 6 -week study, there was a significant increase in the urinary protein excretion. Changes in heparan sulfate glycosaminoglycan chains were not investigated in the present study. However, according to the results of previous studies a loss of heparan sulfate proteoglycans in the GBM contributes to proteinuria in glomerular diseases including DN [21, 22]. Therefore the suludexide as heparanase inhibitor might have prevented diabetic nephropathy - associated heparan sulfate loss and reduced proteinuria.

Although some previous studies showed that urinary excretion of sulfated polysaccharides were reduced in diabetic rats [23, 24], the present study could not confirm these findings and our results confirmed that urinary GAG excretion was elevated in the non-treated diabetic rats as shown before in diabetic rats by Reddi $[18,25]$ and in diabetic humans by McAuliffe $[26,27]$ and PopA [27]. The studies mentioned above suggested that this decreased urinary glycosaminoglycan concentration could be related to the decreased glycosaminoglycan contents of the kidneys and other tissues in diabetic animals, as reported by Cechowska-Pasko et al. [28], but it could also be due to altered renal permselectivity to negatively charged molecules. As noted, the results of the present study showed that urinary GAG excretion was elevated in the non-treated diabetic rats and sulodexide (haparanase inhibitor) treatment significantly decreased urinary GAG excretion in DN rats compared with the non-treated DN rats. Urinary GAG excretion was significantly correlated with the protein excretion in all the study groups. Since urinary GAG levels correlated with urinary protein concentration, it is possible that the glomerular basement membrane is leaky for these substances. Decreased GAG level in GBM of kidney results in its leakiness leading to proteinuria [29]. The decrease in GAG levels in the kidney could be due to decreased synthesis or increased degradation. How decreased glomerular synthesis caused increased urine excretion of GAGs is not clearly understood. An increase in heparanase activity, an enzyme which is known to degrade GAGs such as heparin sulfate, was found to increase in glomeruli of diabetic rats [30]. As previously mentioned, increase in urinary GAGs during diabetes negatively correlated whit kidney GAGs. This could reflect increased GAG degradation as well as changes in kidney GAGs and their increased urine excretion . Diabetic nephropathy kidney tissue showed progressive glomerular and tubular fibrosis as a final pathway, which eventually affects all substructures in the kidney. The present study provided evidence that the inhibition of the heparanase was functionally significant, which resulted in reduced proteinuria, ECM accumulation and collagen deposition in the glomeruli in vivo.

In conclusion, the sulodexide (haparanase inhibitor) treatment reduced urinary GAG and protein excretion, prevented GBM thickness and collagen deposition and probably protected from the loss of GBM anionic content in DN rats. Preservation of GAG and anionic charges of the GBM seems to be one of the mechanisms by which sulodexide ameliorates the renal histological and functional changes in diabetic rats.

\section{Acknowledgment \\ This study was supported by the Urmia University of Medi- cal Sciences (UMSU) (grant No. 2013). The authors also thank the experienced personnel of the Nephrology and Kidney Transplant Research Center of the UMSU.}

\section{Conflict of interests}

The authors declare that they have no competing interests

\section{REFERENCES}

1. Zemin C, EC. M. Pathogenesis of diabetic nephropathy. J Diabetes Invest 2011;2:243-7.

2. Fard AA, Abbasnezhad P, Makhdomi K, et al. Association of Serum Prolactin Concentrations with Renal Failure in Diabetic Patients. Romanian Journal of Diabetes Nutrition and Metabolic Diseases. 2017;24(3):179-85.

3. Ibrahim HN, TH. H. Diabetic nephropathy. J Am Soc Nephrol. 1997;8:487-93.

4. Atkins R, Zimmet PI-I. World Kidney Day Steering Committee: Diabetic kidney disease: Act now or pay later. Nat Rev Nephrol. 2010;6:134-6.

5. Whitelock JM, Murdoch AD, lozzo RV, Underwood PA. The degradation of human endothelial cell-derived perlecan and release of bound basic fibroblast growth factor by stromelysin, collagenase, plasmin, and heparanases. J Biol Chem. 1996;271(17):10079-86.

6. Kato M, Wang H, Kainulainen V, et al. Physiological degradation converts the soluble syndecan-1 ectodomain from an inhibitor to a potent activator of FGF-2. Nature medicine. 1998;4(6):691-7. 
7. Makino H, Ikeda S, Haramoto T, Ota Z. Heparan sulfate proteoglycans are lost in patients with diabetic nephropathy. Nephron. 1992;61(4):415-21.

8. Kanwar YS, Farquhar MG. Presence of heparan sulfate in the glomerular basement membrane. Proceedings of the National Academy of Sciences. 1979;76(3):1303-7.

9. Shafat I, Ilan N, Zoabi S, Vlodavsky I, Nakhoul F. Heparanase levels are elevated in the urine and plasma of type 2 diabetes patients and associate with blood glucose levels. PloS one. 2011;6(2):e17312.

10. Rops AL, Van den Hoven MJ, Veldman BA, et al. Urinary heparanase activity in patients with Type 1 and Type 2 diabetes. Nephrology Dialysis Transplantation. 2012;27(7):2853-61.

11. Rosenzweig L, Kanwar Y. Removal of sulfated (heparan sulfate) or nonsulfated (hyaluronic acid) glycosaminoglycans results in increased permeability of the glomerular basement membrane to $125 \mathrm{I}$-bovine serum albumin. Lab Invest. 1982;47(2):177-84.

12. Kolset $S$, Reinholt F, Jenssen T. Diabetic nephropathy and extracellular matrix. J Histochem Cytochem. 2012;60(12):976-86.

13. Lauver DA, Lucchesi BR. Sulodexide: a renewed interest in this glycosaminoglycan. Cardiovascular Therapeutics. 2006;24(3-4):214-26.

14. Harenberg J. Review of pharmacodynamics, pharmacokinetics, and therapeutic properites of sulodexide. Med Res Rev. 1998;18(1):1-20

15. Dedov I, Shestakova M, Vorontzov A, Palazzini E. A randomized, controlled study of sulodexide therapy for the treatment of diabetic nephropathy. Nephrology, dialysis, transplantation: official publication of the European Dialysis and Transplant Association-European Renal Association. 1997;12(11):2295-300.

16. Skrha J, Perusicová J, Pont'uch P, Oksa A. Glycosaminoglycan sulodexide decreases albuminuria in diabetic patients. Diabetes Res Clin Pract. 1997;38(1):25-31.

17. Lewis EJ, Lewis JB, Greene T, et al. Sulodexide for kidney protection in type 2 diabetes patients with microalbuminuria: a randomized controlled trial. Am J Kid Dis,2011;58(5):729-36.

18. Reddi AS, Ramamurti R, Miller M, et al. Enalapril improves albuminuria by preventing glomerular loss of heparan sulfate in diabetic rats. Biochem Med Metab Biol. 1991;45(119-131).
19. Sugimoto H, Grahovac G, Zeisberg M, Kalluri R. Renal fibrosis and glomerulosclerosis in a new mouse model of diabetic nephropathy and its regression by bone morphogenic protein-7 and advanced glycation end product inhibitors. Diabetes. 2007;56(7):1825-33.

20. Brosius FC, Alpers CE, Bottinger EP, et al. Mouse models of diabetic nephropathy. J Am Soc Nephrol. 2009;20(12):2503-12.

21. Conde-Knape K. Heparan sulfate proteoglycans in experimental models of diabetes: a role for perlecan in diabetes complications. Diabetes Metab Res Rev. 2001;17(6):412-21.

22. Vanden Born J, Van Kraats AA, Hill S, et al. Vessel wall heparan sulfate and transcapillary passage of albumin in experimental diabetes in the rat. Nephrol Dial Transplant. 1997;12(2):27-31.

23. De Lima CR, Aguiar JA, Michelacci YM. Reduced urinary excretion of sulfated polysaccharides in diabetic rats. Biochim Biophys Acta.2005;1741(1-2):30-41.

24. Cadaval RA, Kohlman O, Michelacci YM. Urinary excretion of glycosaminoglycans and albumin in experimental diabetes mellitus. Glycobiology. 2000;10(2):185-92.

25. Yavuz DG, Ersöz HO, Tuncel M et al. Effects of Aminoguanidine on Glomerular Basement Membrane Thickness and Anionic Charge in a Diabetic Rat Model. Int Jnl Experimental Diab Res. 2001;2:225-32.

26. McAuliffe A, Fisher E, McLennan S, et al. Urinary glycosaminoglycan excretion in NIDDM subjects: Its relationship to albuminura. Diabetic medicine. 1996;13(8):758-63.

27. Popławska-Kita A, Mierzejewska-Iwanowska B, Szelachowska $\mathrm{M}$ et al. Glycosaminoglycans urinary excretion as a marker of the early stages of diabetic nephropathy and the disease progression. Diabetes Metab Res Rev 2008;24(4):310-7.

28. Cechowska-Pasko M, Pałka J, Bańkowski E. Decrease in the glycosaminoglycan content in the skin of diabetic rats. The role of IGF-I, IGF-binding proteins and proteolytic activity. Mol Cell biochem. 1996;154(1):1-8.

29. Juretić D, Krajnović V, Lukac-Bajalo J. Altered distribution of urinary glycosaminoglycans in diabetic subjects. Acta diabetol. 2002;39(3):123-8.

30. Harvey SJ, Miner JH. Breaking down the barrier: evidence against a role for heparan sulfate in glomerular permselectivity. J Am Soc Nephrology. 2007. 